\title{
Species-resolved sequencing of low- biomass or degraded microbiomes using 2bRAD-M
}

Zheng Sun ${ }^{1,2,3 \dagger}$, Shi Huang ${ }^{1,3,4,5^{*}+}$, Pengfei Zhu ${ }^{1,3}$, Lam Tzehau ${ }^{6}$, Helen Zhao ${ }^{6}$, Jia Lv ${ }^{7}$, Rongchao Zhang ${ }^{8}$, Lisha Zhou ${ }^{8}$, Qianya Niü ${ }^{8}$, Xiuping Wang ${ }^{8}$, Meng Zhang ${ }^{9}$, Gongchao Jing ${ }^{1,3}$, Zhenmin Bao ${ }^{7,10,11}$, Jiquan Liu', Shi Wang ${ }^{7,10,11^{*}}$ and Jian $X u^{1,3,10^{*}}$ (D)

\author{
*Correspondence: huangshi@ \\ qibebt.ac.cn; swang@ouc.edu.cn; \\ xujian@qibebt.ac.cn \\ †Zheng Sun and Shi Huang \\ contributed equally to this work. \\ ${ }^{1}$ Single-Cell Center, CAS Key \\ Laboratory of Biofuels and \\ Shandong Key Laboratory of Energy \\ Genetics, Qingdao Institute of \\ BioEnergy and Bioprocess \\ Technology, Chinese Academy of \\ Sciences, Qingdao, China \\ ${ }^{7}$ Sars-Fang Centre \& MOE Key \\ Laboratory of Marine Genetics and \\ Breeding, Ocean University of \\ China, Qingdao, Qingdao, China \\ Full list of author information is \\ available at the end of the article
}

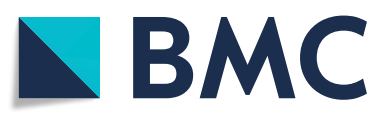

\section{Introduction}

Metagenome sequencing, widely used to derive the taxonomic profile of microbiome, typically adopts two strategies that target (i) amplicons of phylogenetic "marker genes" (e.g., 16S rRNA for bacteria and archaea, and 18S rRNA or internal transcribed spacer (ITS) for fungi) or (ii) the whole genomes (whole metagenome shotgun; WMS). Although less costly, marker gene analyses can be limited in taxonomic resolution (i.e., at the genus level) and susceptible to PCR bias in composition and abundance estimates [1]; moreover, they are usually unable to capture a landscape-like view that includes bacteria, archaea, fungi, and virus due to the lack of universal primers. In contrast, by sequencing the total DNA, WMS can resolve species- or strain-level taxonomy and offers a landscape-like view that includes all domains of organisms [2, 3]. However, although $1 \mathrm{ng}$ is applicable via certain specialized kits [4], WMS usually requires a high amount of DNA as the starting material ( $\geq 50 \mathrm{ng}$ preferred, $20 \mathrm{ng}$ at a minimum) and moreover is not efficient in tackling DNA samples that are low in biomass, heavily degraded, or dominated by host DNA [1, 4]. Furthermore, WMS is typically much more costly, due to the much higher sequencing volume required for covering the

(c) The Author(s). 2022 Open Access This article is licensed under a Creative Commons Attribution 4.0 International License, which permits use, sharing, adaptation, distribution and reproduction in any medium or format, as long as you give appropriate credit to the original author(s) and the source, provide a link to the Creative Commons licence, and indicate if changes were made. The images or other third party material in this article are included in the article's Creative Commons licence, unless indicated otherwise in a credit line to the material. If material is not included in the article's Creative Commons licence and your intended use is not permitted by statutory regulation or exceeds the permitted use, you will need to obtain permission directly from the copyright holder. To view a copy of this licence, visit http//creativecommons.org/licenses/by/4.0/. The Creative Commons Public Domain Dedication waiver (http://creativecommons.org/publicdomain/zero/1.0/) applies to the data made available in this article, unless otherwise stated in a credit line to the data. 
whole genome (instead of just the marker gene). Therefore, new methods should be developed that cost-efficiently produce accurate, species-resolution, landscape-like taxonomic profiles for challenging samples like low-biomass, high host-contaminated, and degraded microbiomes.

Restriction site-associated DNA sequencing (RADseq) that utilizes restriction enzymes to digest genomic DNA from a broad range of organisms and sequences only the digested fragments has been applied to genotype variable genetic markers as well as for microbial species detection $[5,6]$. Although RADseq has demonstrated the ability to produce species-resolution microbiome profiles with lower costs [7-10], the large size variation of DNA fragments after enzyme digestion results in a high bias in PCR-based amplification and thus the low fidelity of reconstructed taxonomic profile [11, 12]. Moreover, RADseq has not been thoroughly and systematically benchmarked against marker gene-based or WMS approaches [7-10]. Therefore, it remains unclear whether RADseq can provide accurate, landscape-like taxonomic profiles for low-biomass and severely degraded microbiome samples.

To address these challenges, here, we proposed the "2bRAD sequencing for Microbiome" (2bRAD-M) method that utilizes Type IIB restriction enzymes to produce exclusively iso-length DNA fragments of $25-33 \mathrm{bp}$ (depending on the selection of enzymes) for sequencing [13-15]. This approach reduces the bias in PCR-based fragment amplification and thus ensures the high fidelity of the taxonomic profile. This offers significant benefits especially for low-biomass, heavily contaminated, or degraded microbial DNA which requires more PCR cycles. Tests on simulated datasets, mock, and actual microbiome samples illustrate that $2 \mathrm{bRAD}-\mathrm{M}$, by sequencing just about $1 \%$ of genomes, accurately generates species-level taxonomic profiles for challenging samples: (i) of merely 1 pg total DNA, (ii) of 99\% host DNA contamination, or (iii) consisting of highly degraded fragments just 50-bp long. For real stool, skin, and environment-surface samples, it accurately reconstructs a comprehensive, speciesresolution profile of bacteria, archaea, and fungi. Furthermore, microbiome in the formalin-fixed paraffin-embedded (FFPE) tissue samples which were otherwise recalcitrant to sequencing can now be analyzed, and a species-resolved classifier discriminated the healthy tissue, pre-invasive cancer, and invasive cancer with $91.1 \%$ accuracy. The ability to profile low-biomass microbiomes at the species level is pivotal to expanding the boundary of the known microbial world.

\section{Results}

The principle and workflow of 2bRAD-M

The principle and appealing features of 2bRAD-M are as follows (Fig. 1): (i) reliable enzyme-digested sequence tags can be derived that are specific to high-resolution taxa (e.g., species or strain) yet universally applicable for a broad range or all of bacterial, archaeal, and fungal genomes; (ii) these taxa-specific, iso-length sequence tags can be evenly amplified and sequenced; and (iii) the tag sequences can be mapped to reference genomes to reconstruct faithfully the taxonomic composition.

Specifically, the experimental workflow has two steps: (i) BcgI (a commercially available Type IIB restriction enzyme) is used, as an example, to digest total genomic DNA extracted from microbiome samples. BcgI recognizes the sequence of $\mathrm{CGA}^{-\mathrm{N}_{6}}$-TGC in 


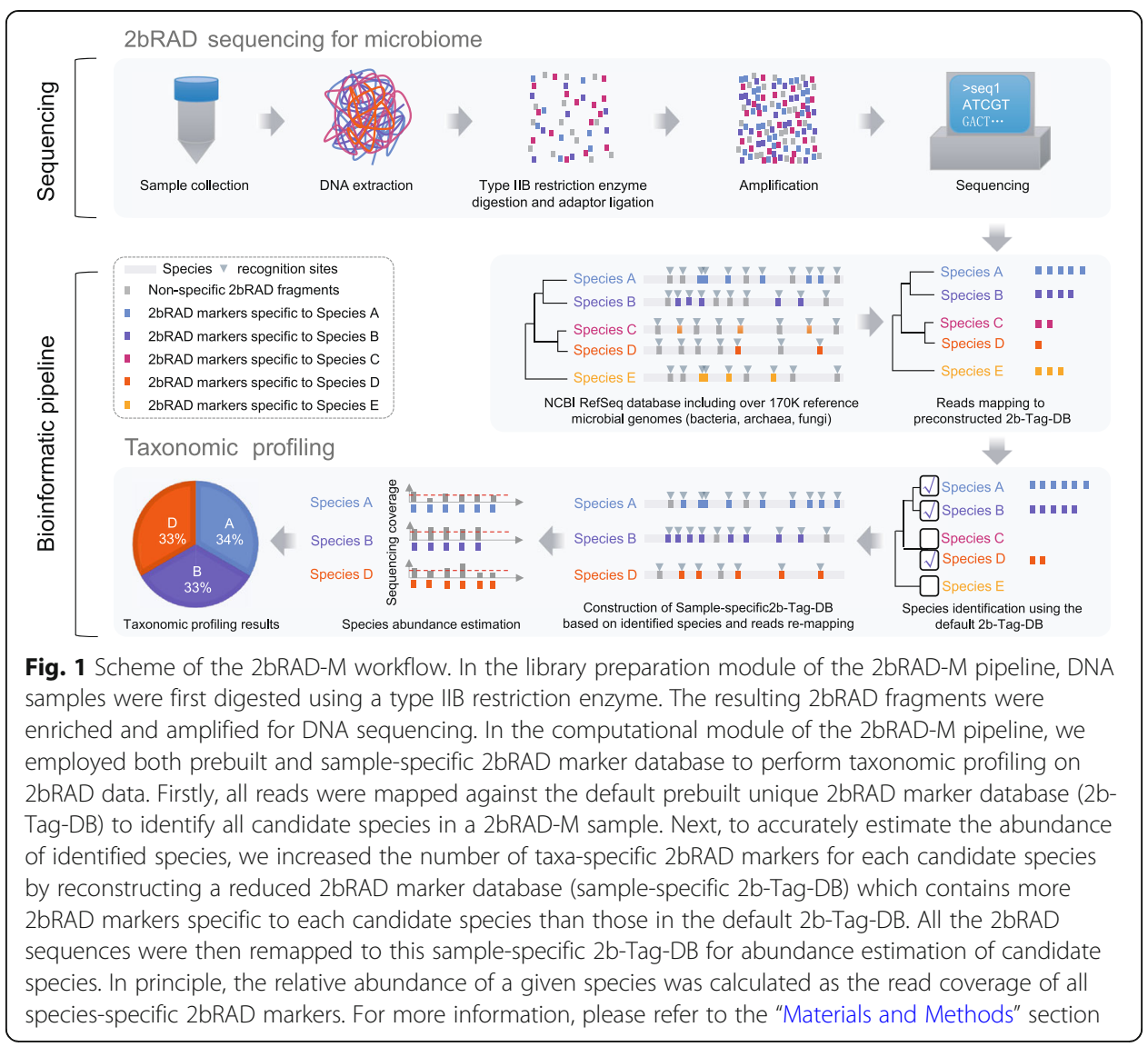

the genomic DNA and cleaves on both upstream (12-10 bp) and downstream (10-12 bp) of this signature [13], producing short and iso-length DNA (32 bp without sticky ends) across all loci $[14,15]$. (ii) These so-called 2bRAD fragments are ligated to adaptors, amplified, and then sequenced.

In the computational workflow, the foundation is a unique $2 \mathrm{bRAD}$ tag database (" $2 \mathrm{~b}$ Tag-DB"), which contains taxa-specific 2bRAD tags identified from all the sequenced bacteria, fungi, and archaea genomes. Mapping the 2bRAD reads against 2b-Tag-DB thus identifies the presence of species in a sample. Subsequently, to estimate the relative abundance of the identified taxa, the mean read coverage of all 2bRAD tags specific to each taxon is derived. To improve the utilization rate of reads and classification accuracy, a secondary, sample-specific 2b-Tag-DB was dynamically derived from only those candidate taxa identified in a particular sample, which produces more speciesspecific $2 \mathrm{bRAD}$ tags than the original $2 \mathrm{~b}$-Tag-DB and results in more accurate modeling of relative abundance of taxa.

The feasibility of 2bRAD-M for microbiome analysis via in silico simulation

To identify the taxa-specific tags, we first downloaded 173,165 microbial genomes (171,927 bacteria, 293 fungi, and 945 archaea; representing 26,163 species) from NCBI RefSeq (Oct 2019) to create a 2b-Tag-DB via in silico restriction digestion of these genomes using BcgI restriction enzyme [13]. This yields an average of 3010 iso-length 
(32 bp) 2bRAD tags per genome, and a total of 114,132,487 BcgI-digested unique species-specific 2bRAD tags that are of single-copy within a genome (average 1194 per species genome; Additional file 1: Supplemental Methods). Besides BcgI, speciesspecific $2 \mathrm{bRAD}$ tags (unique 2bRAD tags) were also identified for each of the other 15 Type IIB enzymes (AlfI, AloI, BaeI, BplI, BsaXI, BslFI, Bsp24I, CjeI, CjePI, CspCI, FalI, HaeIV, Hin4I, PpiI, and PsrI; Additional file 1: Table S1, Fig. S1a) to establish the usability of all Type IIB enzymes for 2bRAD-M. Notably, 2bRAD tags are detected in all genomes regardless of the Type IIB restriction enzyme used (Additional file 1: Fig. S1b). Moreover, the number of 2bRAD tags within a genome and their GC content are highly consistent with the length and the GC\% of genomes $(r>0.98)$ (Additional file 1 : Fig. S2) for all the 16 Type IIB restriction enzymes, suggesting unbiased and broadly applicable representation of the microbial genomes by these tags.

To test whether these species-specific tags enable detection and abundance profiling of all known species in a community, a simulated 50-species microbiome was generated (one genome per species; randomly selected from RefSeq; Additional file 1: Table S2) and profiled using the default $2 \mathrm{~b}-\mathrm{Tag}-\mathrm{DB}$ and sample-specific $2 \mathrm{~b}-\mathrm{Tag}-\mathrm{DB}$ for each of the 16 Type IIB restriction enzymes. Performance of 2bRAD-M in species detection was assessed via precision and recall (with relative abundance threshold of 0.0001), while that of species abundance evaluated via L2 similarity score (a metric of similarity adapted from L2 distance [16]), by comparing to the ground truth (Fig. 2a). Precision, recall, and L2 similarity of the taxonomic profiling are all remarkably high (average for the 16 enzymes - precision $=98.0 \%$, recall $=98.0 \%$, L2 similarity $=96.9 \%$, and this is achieved with an average genome coverage of $1.50 \%$ among the 50 selected genomes (Fig. 2a, Additional file 1: Fig. S3).

The accuracy and computational efficiency of 2bRAD-M were assessed by comparison with highly cited WMS profiling tools such as Kraken2 [17], Bracken [18], mOTUs2 [19], and MetaPhlAn2 [20], using the in silico simulated data of 25 microbial communities for benchmarking [21] (2bRAD-M applied BcgI-derived species-unique markers to profile the simulated data, while the others produced the abundance profiles from simulated pair-end 150-bp WMS reads; the "Materials and Methods" section). In terms of average Shannon similarity, Bray-Curtis similarity, L2 similarity (accuracy metrics evaluated based on the ground truth), precision, and recall, 2bRAD-M showed a level of $0.998,0.97,0.98,0.89$, and 0.98, respectively, which either outperformed or are equivalent to others (Fig. 2b). As for database storage and memory use, 2bRAD-M requires $<10-G B$ disk space to store the reference marker database and a relatively low RAM of $30 \mathrm{~GB}$ (equivalent to a desktop computer) as compared to Centrifuge, Kraken2, and Bracken (Fig. 2b). Thus, the 2bRAD-M bioinformatic pipeline can provide accurate profiling results with high computational efficiency.

\section{High reproducibility and sensitivity of $2 \mathrm{bRAD}-\mathrm{M}$ under challenging conditions}

To assess 2bRAD-M ability to handle low-biomass, highly degraded, or heavily contaminated samples, we first constructed a mock community (Mock-CAS) consisting of five prevalent oral or gut bacterial species in equal proportion. With this mock community stock, three series of samples were prepared: (i) "LoA" (low amount)-samples with total DNA amounts from $50 \mathrm{ng}, 20 \mathrm{ng}, 10 \mathrm{ng}, 1 \mathrm{ng}, 100 \mathrm{pg}, 10 \mathrm{pg}$ to only $1 \mathrm{pg}$; (ii) "HiD" 


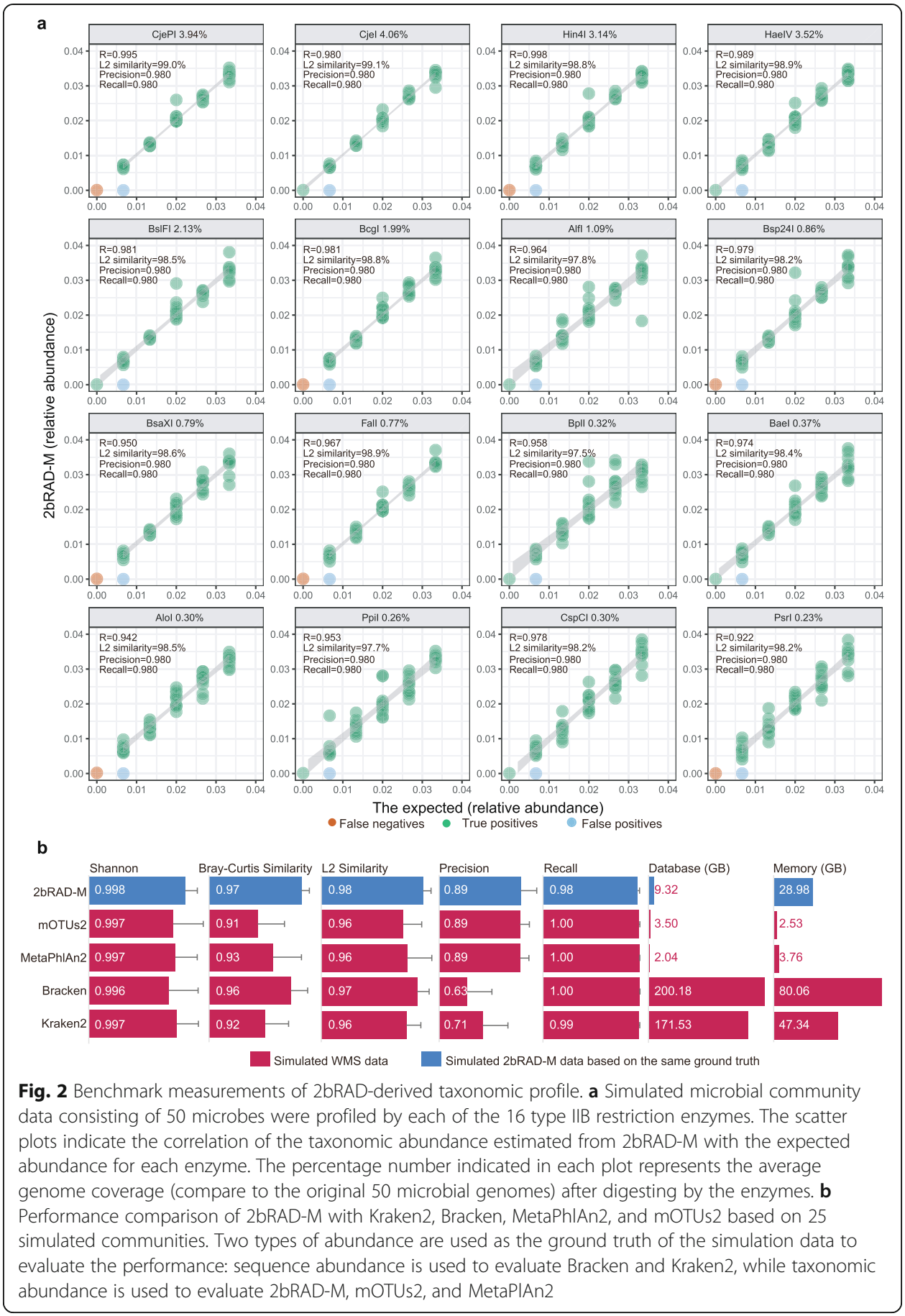

(high degradation) - 100-ng degraded DNA samples where the DNA mixture was randomly sheared by DNAse I into fragments of mostly $150 \mathrm{bp}$ and $50 \mathrm{bp}$ in length; and (iii) "HoC" (host contamination) - 100-ng samples spiked with human DNA to simulate $90 \%$ or $99 \%$ contamination by host genome. Three technical replicates were included for each of the LoA $(n=7 * 3)$, HiD $(n=2 * 3)$, and HoC $(n=2 * 3)$ groups. Each of the 33 samples from the three series was then sequenced by $2 \mathrm{bRAD}-\mathrm{M}$. In addition, a sample 
of 100-ng DNA was then prepared from the mock community stock and sequenced by WMS to be referenced as the positive control (WMS of $50 \mathrm{ng}$ or lower DNA failed).

To compare the results and minimize performance bias introduced by bioinformatic pipelines, we employed Centrifuge [22] to map both WMS (150-bp) and 2bRAD-M (32-bp) generated reads to the reference microbial genomes for taxonomic abundance estimation. For 2bRAD-M, tags from the actual data of each sample covered avg. 97.1\% in silico predicted tags from the five genomes, indicating high consistency between observed and expected 2bRAD tags (which is key to accurate and reliable taxonomic profiling). Moreover, for each sample, the 2bRAD-M results are highly consistent among the three biological replicates (avg. L2 similarity: 95.4\%; Fig. 3a).

Notably, for the LoA, HiD, and $\mathrm{HoC}$ groups, the average L2 similarity between 2bRAD-M profiles and the reference positive control is $88.9 \%, 84.6 \%$, and $89.6 \%$,

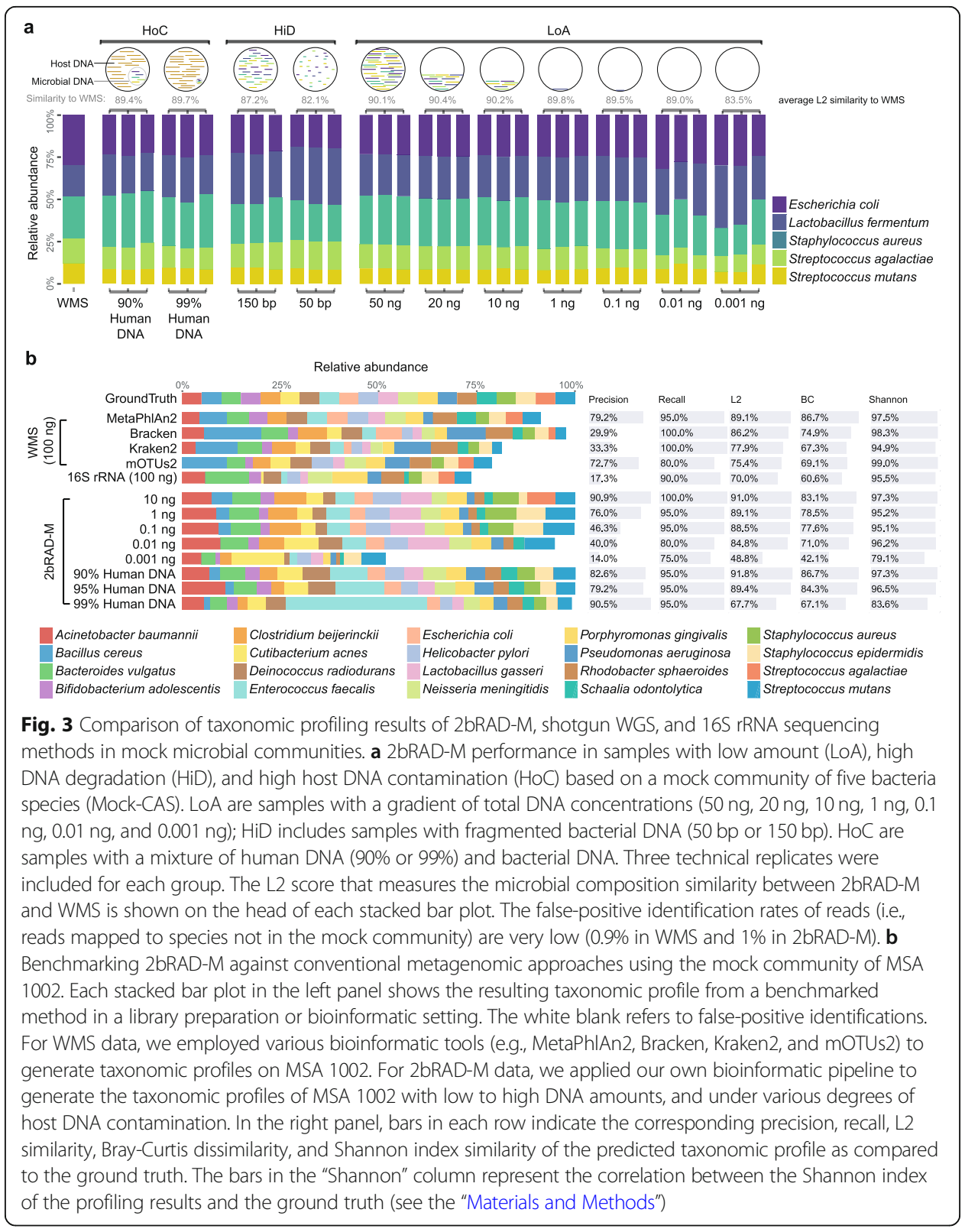


respectively, with the global average being $88.2 \%$ (ranging from 82.1 to $90.4 \%$; Fig. 3a). These observations are consistent across the technical replicates, indicating high reproducibility of 2bRAD-M. Specifically, in the LoA group, the L2 similarity of the 1-pg sample can still realize a respectable $83.5 \%$ as compared to $90.1 \%$ for the 50 -ng sample (Fig. 3a). This suggests that 2bRAD-M offers high sensitivity and stable performance in low-biomass samples over a broad DNA-amount range (from $50 \mathrm{ng}$ to $1 \mathrm{pg}$ ). In the HiD group, the L2 similarity is $87.2 \%$ and $82.1 \%$ for the 150 -bp and 50 -bp samples, respectively, indicating DNA degradation did not have a large negative effect and the $2 \mathrm{~b}$ RAD-M can effectively accommodate severe DNA degradation while providing reliable results. For the HoC group, the L2 similarity is $89.4 \%$ in the $90 \%$-host-contaminated samples and $89.7 \%$ in 99\%-host-contaminated samples (in addition, a certain degree of enrichment of microbiome-originated reads versus host-originated reads is apparent under HoC; Additional file 1: Table S3), suggesting 2bRAD-M's ability to provide reliable microbial profiling in the presence of host DNA contamination.

To further probe the capability of $2 \mathrm{bRAD}-\mathrm{M}$ in profiling a highly complex microbial community at low-biomass setting, we used ATCC MSA 1002, a standard, commercially available mock community consisting of 20 bacterial species (from 18 genera) with at equal DNA abundance among species [23]. Samples with total DNA amount ranging from $10 \mathrm{ng}, 1 \mathrm{ng}, 100 \mathrm{pg}, 10 \mathrm{pg}$, and $1 \mathrm{pg}$ and DNA host contamination from 90\%, 95\%, and 99\% were prepared from this mock community for 2bRAD-M profiling. As a reference control, a 100-ng sample was profiled with the 16S-rRNA and WMS approaches (the 1-pg 10-ng samples failed). The precision for $2 \mathrm{bRAD}-\mathrm{M}$ is $90.9 \%, 76.0 \%$, $46.3 \%, 40.0 \%, 14.0 \%, 82.6 \%, 79.2 \%$, and $90.5 \%$ for the $10 \mathrm{ng}, 1 \mathrm{ng}, 100 \mathrm{pg}, 10 \mathrm{pg}, 1 \mathrm{pg}$, 90\% host DNA, 95\% host DNA, and 99\% host DNA samples, respectively. This is in contrast to the $53.7 \%$ (average number of different profilers) for WMS-100 ng and the $17.3 \%$ for 16S-rRNA-100 ng (Fig. 3b). Therefore, 2bRAD-M offers much lower false positives in detecting the species than 16S-rRNA and WMS. As for recall, 2bRAD-M is $100.0 \%$, 95.0\%, 95.0\%, 80.0\%, 75.0\%, and 95\% for $10 \mathrm{ng}, 1 \mathrm{ng}, 100 \mathrm{pg}, 10 \mathrm{pg}, 1 \mathrm{pg}$, and host DNA-contaminated samples, respectively, as compared to the $93.7 \%$ for WMS$100 \mathrm{ng}$ and the $90.0 \%$ for $16 \mathrm{~S}-\mathrm{rRNA}-100 \mathrm{ng}$. Therefore, 2bRAD-M offers a comparable level of sensitivity to WMS and 16S-rRNA when starting with down to $100 \mathrm{pg}$ of DNA. Moreover, the L2 similarity for 2bRAD-M is $91.0 \%, 89.1 \%, 88.5 \%, 84.8 \%, 48.5 \%, 91.8 \%$, $89.4 \%$, and $67.7 \%$ for $10 \mathrm{ng}, 1 \mathrm{ng}, 0.1 \mathrm{ng}, 10 \mathrm{pg}, 1 \mathrm{pg}, 90 \%$ host DNA, $95 \%$ host DNA, and $99 \%$ host DNA samples, respectively, as compared to $82.1 \%$ for WMS-100 ng and $70.0 \%$ for $16 \mathrm{~S}-\mathrm{rRNA}-100 \mathrm{ng}$. As for alpha diversity (Shannon index) and beta diversity (Bray-Curtis similarity), profiling results of the low-biomass samples by 2bRAD-M are comparable to the corresponding high biomass samples by WMS and $16 \mathrm{~S}$ as well (alpha diversity: averagely $92.5 \%$ by 2 bRAD-M, $97.4 \%$ by WMS, and $95.5 \%$ by $16 \mathrm{~S}$; beta diversity: averagely $73.8 \%$ by 2 bRAD-M, $74.6 \%$ by WMS, and $60.6 \%$ by $16 \mathrm{~S}$ ). Taken as a whole, these results demonstrated 2bRAD-M's ability to profile complex microbiota with a high level of sensitivity and specificity.

2bRAD-M enables cost-effective deep microbiome profiling of real samples (fecal)

To assess the performance of $2 \mathrm{bRAD}-\mathrm{M}$ on real samples, we performed and compared 2bRAD-M and 16S rRNA sequencing on human fecal samples $(n=3)$. In addition, each 
sample was also subjected to ultra-deep WMS sequencing (mean of 437 million reads or $239.73 \mathrm{~Gb}$ per sample) with the resultant taxonomic profiles used as evaluation reference [24].

Taxonomic profiles from 2bRAD-M are compared with those from 16S rRNA sequencing (Fig. 4a-c) and WMS (Fig. 4d-f; Additional file 1: Supplemental Methods). Specifically, at the genus level, results of 2bRAD-M and 16S rRNA are highly consistent (mean Pearson correlation $R=0.997$ and mean L2 similarity L2 $=92.0 \%$ ), and avg. $95.27 \%$ of genus identified by $16 \mathrm{~S}$ were also detected by 2 bRAD-M (Additional file 1 : Table S4). As for the species level, to perform the comparison in a fair manner, we extracted $2 \mathrm{bRAD}$ reads from WMS data and then used these WMS-originated 2bRAD reads as input for the $2 \mathrm{bRAD}-\mathrm{M}$ computational pipeline; then, we found that WMSoriginated $2 \mathrm{bRAD}$ reads and real $2 \mathrm{bRAD}$ sequencing data are also concordant in species-level profiling results, as evidenced by a high Pearson correlation $(R=0.99)$ and high L2 similarity (up to $97.8 \%$ ). Moreover, only $0.40 \%$ of the taxa in WMS were not identified in 2bRAD-M (Additional file 1: Table S5). Thus, 2bRAD-M can produce highly complete and accurate species-level profiles that are equivalent to WMS.

One determinant of quality (and cost) of taxonomic profiling is sequencing depth [25]. To test how it influences 2bRAD-M performance, the fecal sequencing datasets were subsampled to various depths for 2bRAD-M (average 13.5 million reads per sample, subsampled at $0.1,1,2,3,5$ to 13.5 million sequences per sample) or WMS (subsampled at $0.5,10,20,30,40,50,100$, and 200 million sequences per sample since one $2 \mathrm{~b}$ read can be identified and fetched from about 15 WMS reads) respectively. Then, we asked how many $2 \mathrm{bRAD}$ sequences are required to accurately quantify key ecological metrics such as alpha diversity (Shannon index), beta diversity (Bray-Curtis), and species compositions (species richness). In each of the fecal samples, with 20-40

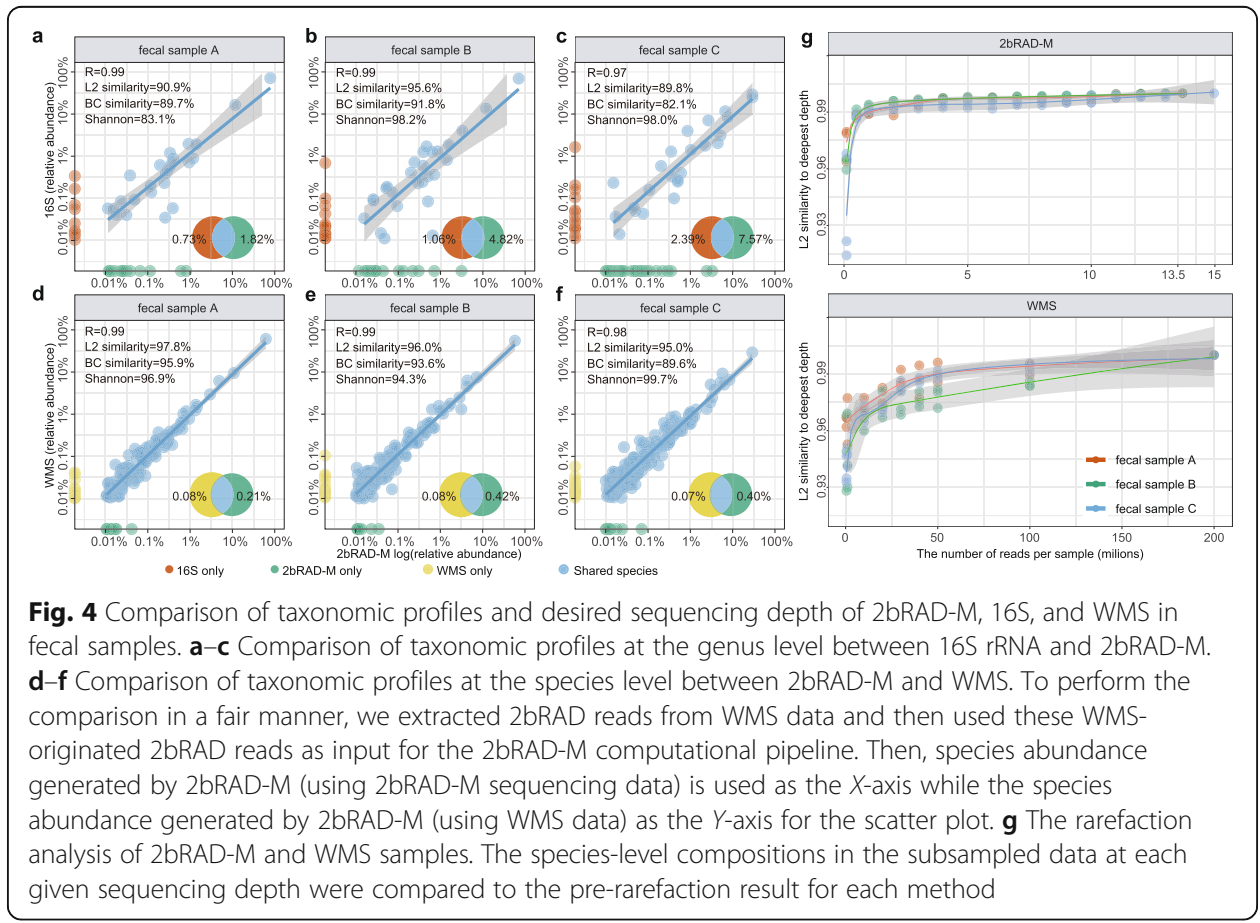


million reads per sample, WMS identified averagely $170-182$ species, but the Shannon diversity and species abundance estimates are far from saturation (i.e., reaching the values derived by sequencing $\sim 400$ million reads). In contrast, with just 3-4 million reads per sample, 2bRAD-M identified 173-188 species in each fecal samples and yield Shannon diversity and species abundance estimates comparable with those from the deepest ( 13.5 million) read depth (Additional file 1: Fig. S4).

To evaluate the optimal sequencing depth of 2bRAD-M for species-level taxonomic profiling, we evaluated the similarity of taxonomic profiles derived at each reduced sequencing depth to its original taxonomic profile using the L2 similarity score. For 2bRAD-M, a sequencing depth of over 0.5 million reads per sample can achieve a L2 similarity score of 98.9\% (1 million for 99.1\%), which however requires many more (at least about 50 million) reads per sample in WMS (Fig. 4g). Overall, with $2 \sim 3$ million reads (i.e., $60 \mathrm{Mb}$ of sequencing data) per sample, 2bRAD-M can generate consistent, accurate, and stable alpha diversity estimates and taxonomic profile at the species level.

\section{2bRAD-M enables species-resolved analysis of low-biomass skin, home, and car samples}

To assess 2bRAD-M performance on actual low-biomass samples, we collected samples from the human skin surface (underarm, $n=20$; Additional file 1: Table S6) for 16S-rRNA and 2bRAD-M analysis (WMS was aborted due to too-low DNA amounts for library construction). To gauge the potential impact of contaminating DNA (introduced from regents, in workflow, etc.) in the low-biomass samples [26], MSA 1002 was included as a control. MSA 1002 profiling revealed the expected microbial community profile while unforeseen microbes were absent which indicates minimal contamination. Between 16S rRNA and 2bRAD-M, a high degree of consistency in taxonomic profiles at the genus level was observed, with avg. L2 similarity of $81.1 \%$ (Table 1; Additional file 1: Fig. S5). In both methods, Staphylococcus (2bRAD-M: avg. 47.17\%; $16 \mathrm{~S}$ rRNA: avg. 44.43\%) and Corynebacterium (2bRAD-M: avg. 10.38\%; 16S rRNA: avg. 15.06\%) are recognized as dominant microbes, consistent with existing literature [27-29].

We further explored the application of 2bRAD-M, by testing on indoor environmental low-biomass samples, which are of significant health implications due to their contact with humans [30]. Surfaces of floor mat $(n=5)$ and cushion $(n=3)$ in the car and interior surfaces of a house $(n=4)$ were sampled and subjected to both 16S rRNA and 2bRAD-M (similarly, WMS was aborted due to the low DNA amounts; Additional file 1: Table S6). High L2 similarity (average 85.6\% among samples; Additional file 1: Fig. S5) between 2bRAD-M and 16S rRNA was observed, suggesting stable performance of 2bRAD-M for such indoor samples. At the species level (Additional file 2), we identified 4929, 2335, and 1626 taxa for the three niches, respectively, whose dominant bacterial species are highly distinct: (i) floor mats are dominated by Kocuria rosea (23.23\%), Psychrobacter 1501_2011 (11.44\%), and Acinetobacter johnsonii (10.43\%); (ii) cushions are dominated by Cutibacterium acnes (42.19\%), Lactobacillus delbrueckii (10.62\%), and Ralstonia pickettii (10.14\%); (iii) home surfaces are mainly colonized by Streptococcus mitis (49.64\%), Prevotella copri (39.14\%), and Megamonas funiformis (18.36\%). Rarefaction of sequence depth (e.g., sequenced 2b-tags) via alpha diversity, 
Table 1 High concordance of dominant microbial taxa in 16S rRNA and 2bRAD-M profiling results of 20 underarm samples. The left three columns illustrate the top five genera in 165 profiling results of the 20 underarm samples with average relative abundance and ranking. The right three columns are the corresponding species' average relative abundance and ranks identified by 2bRAD-M

\begin{tabular}{|c|c|c|c|c|c|}
\hline $\begin{array}{l}\text { Top genus in } \\
16 \mathrm{~S}\end{array}$ & $\begin{array}{l}\text { Relative } \\
\text { abundance }\end{array}$ & Rank & $\begin{array}{l}\text { Corresponding species in } \\
\text { 2bRAD-M }\end{array}$ & $\begin{array}{l}\text { Relative } \\
\text { abundance }\end{array}$ & Rank \\
\hline \multirow[t]{6}{*}{ Staphylococcus } & $44.43 \%$ & 1 & Staphylococcus epidermidis & $27.56 \%$ & 1 \\
\hline & & & Staphylococcus hominis & $12.57 \%$ & 2 \\
\hline & & & Staphylococcus capitis & $4.78 \%$ & 5 \\
\hline & & & Staphylococcus haemolyticus & $1.78 \%$ & 11 \\
\hline & & & The other 4 species & $0.48 \%$ & \\
\hline & & & SUM & $47.17 \%$ & \\
\hline \multirow[t]{8}{*}{ Corynebacterium } & $15.06 \%$ & 2 & Corynebacterium ureicelerivorans & $2.58 \%$ & 8 \\
\hline & & & Corynebacterium jeddahense & $2.41 \%$ & 9 \\
\hline & & & Corynebacterium aurimucosum & $1.80 \%$ & 10 \\
\hline & & & Corynebacterium pseudogenitalium & $1.29 \%$ & 15 \\
\hline & & & Corynebacterium kefirresidentii & $1.23 \%$ & 17 \\
\hline & & & $\begin{array}{l}\text { Corynebacterium } \\
\text { tuberculostearicum }\end{array}$ & $1.08 \%$ & 19 \\
\hline & & & The other 47 species & $2.08 \%$ & \\
\hline & & & SUM & $12.47 \%$ & \\
\hline Uruburuella & $5.67 \%$ & 3 & Uruburuella suis & $5.80 \%$ & 4 \\
\hline \multirow[t]{4}{*}{ Enterococcus } & $4.49 \%$ & 4 & Enterococcus devriesei & $1.68 \%$ & 12 \\
\hline & & & Enterococcus dispar & $1.48 \%$ & 13 \\
\hline & & & The other 3 species & $0.45 \%$ & \\
\hline & & & SUM & $3.61 \%$ & \\
\hline \multirow[t]{3}{*}{ Moraxella } & $02.34 \%$ & 5 & Moraxella osloensis & $2.77 \%$ & 7 \\
\hline & & & The other 1 species & $0.04 \%$ & \\
\hline & & & SUM & $2.81 \%$ & \\
\hline SUM & $72.00 \%$ & & SUM & $71.86 \%$ & \\
\hline
\end{tabular}

beta diversity, and species-level compositions support high robustness of 2bRAD-M for such low-biomass indoor samples (Additional file 1: Fig. S6).

Similar to WMS, 2bRAD-M enabled species-level profiles for fungi along with bacteria. In general, the relative abundance of fungi in the underarm and indoor environment samples is extremely low (0.83\%) as compared to bacteria (99.16\%) (Additional file 1: Table S7). Possible reasons for this observation include (i) micro-environments of these samples are not friendly fungal growth [31] and (ii) the relatively small number of available fungi genomes curated in our reference database limits the discovery of the fungi population. Nonetheless, among all the sites, the samples taken from the car cushion are found to harbor the highest amount of fungi (2.33\%) with the surfaces from home being the lowest $(0.06 \%)$. In addition, the 2bRAD-M species-level profile unveils distinctive patterns of fungal composition among the various sites (Fig. 5a). Malassezia restricta and Malassezia globose, known commensals on human skin surface [32], were found as the top abundance fungi at underarm and most of the indoor environmental samples. It was possible that the Malassesia sp. was transferred from humans, given the likely high contact frequency between the skin and these indoor environmental 


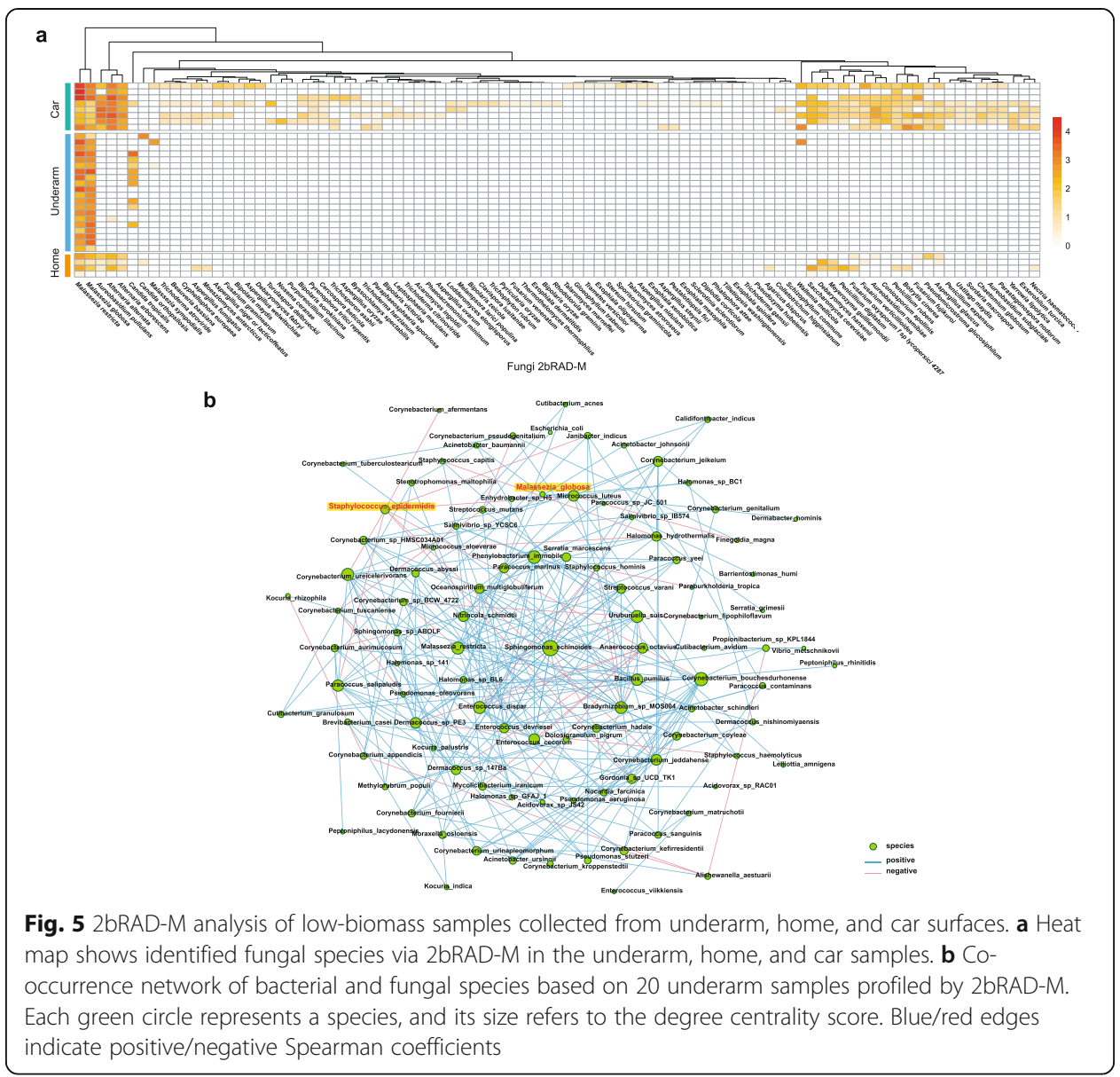

surfaces. Conversely, Alternaria alteranta was mostly found in indoor environments but absent in the underarm which is in agreement with existing reports [33, 34]. Thus, the fungi profiles derived from $2 \mathrm{bRAD}-\mathrm{M}$ are consistent with literature, demonstrating the ability to reliably profile fungi (simultaneously with bacteria) in low-biomass samples.

Taking advantage of 2bRAD-M's capability in profiling bacterial and fungal species simultaneously, an occurrence-network analysis revealed a negative correlation between the human skin commensal yeast of Malassezia globosa (which is associated with Seborrheic Dermatitis [35]) and the aforementioned S. epidermidis (recently proposed as a gatekeeper of healthy skin [36]; Spearman coefficient of -0.569 ; Fig. 5b). Such landscape-like, species-level correlations for low-biomass microbiomes can potentially reveal novel bacteria-fungi interactions.

\section{2bRAD-M enables tumor microbiome profiling from FFPE tissue samples}

Microbiota in human tumor or blood tissues were recently associated with the types, developmental stages, or chemotherapeutic efficiency of cancer [37-41]. Formalinfixed, paraffin-embedded (FFPE) tissue, the gold standard of preserving tumor biopsy specimens [42, 43], represents a vast, irreplaceable historical clinical resource of enormous value for cancer microbiome studies [44]; however, profiling microbiome from 
FFPE tissues has been challenging due to the low microbial biomass, high human DNA contamination, severe DNA damage, and cross-links by chemical modification [45]. To test whether 2bRAD-M can tackle FFPE samples, we started with three pairs of healthy lung tissues from lung adenocarcinoma patients (typical of low microbial biomass), each derived both before (i.e., fresh tissue) and after FFPE (the "Materials and Methods" section). For each of the three pairs of lung tissue samples, a high consistency of microbial profiles derived using 2bRAD-M was reported between pre- and post-FFPE samples (Additional file 1: Fig. S7). These results thus support the ability of 2bRAD-M to accurately reconstruct microbiome structure from FFPE samples.

Next, we collected FFPE cervical tissue samples from 15 healthy controls $(\mathrm{H}), 15$ preinvasive cancerous (PreC; benign), and 15 invasive cancerous patients (InvaC; malignant) and subjected these samples to $2 \mathrm{bRAD}-\mathrm{M}$ sequencing. DNA from the FFPE tissue was extracted from an area of $3 \mathrm{~cm}^{2}$ with $4-\mu \mathrm{m}$ thickness. On average, $25 \mathrm{ng} / \mu \mathrm{l}$ of DNA in each sample was detected as smears of size under $500 \mathrm{bp}$ in agarose gels (Additional file 1: Fig. S8, Table S3), suggesting extremely low concentration and highly fragmented nature of DNA (both human and microbes [45]) in FFPE samples.

The microbiome of the FFPE samples is mostly dominated by bacteria species $(n=$ 243) with minimal fungal species $(n=2$; Additional file 2$)$. The alpha diversity (Shannon and Simpson index) in healthy controls $(\mathrm{H})$ is significantly lower than PreC and InvaC ( $p=0.044$, Kruskal test; Fig. 6a). Among the identified bacterial species (Additional file 1: Fig. S9), samples in the PreC and InvaC groups are significantly enriched with Methyloversatilis discipulorum ( $p$-value $=1.2 \mathrm{e}-5)$, Mycobacterium tuberculosis $(p$-value $=0.004)$, Methyloversatilis universalis $(p$-value $=1.8 \mathrm{e}-5)$, Ferrovibrio
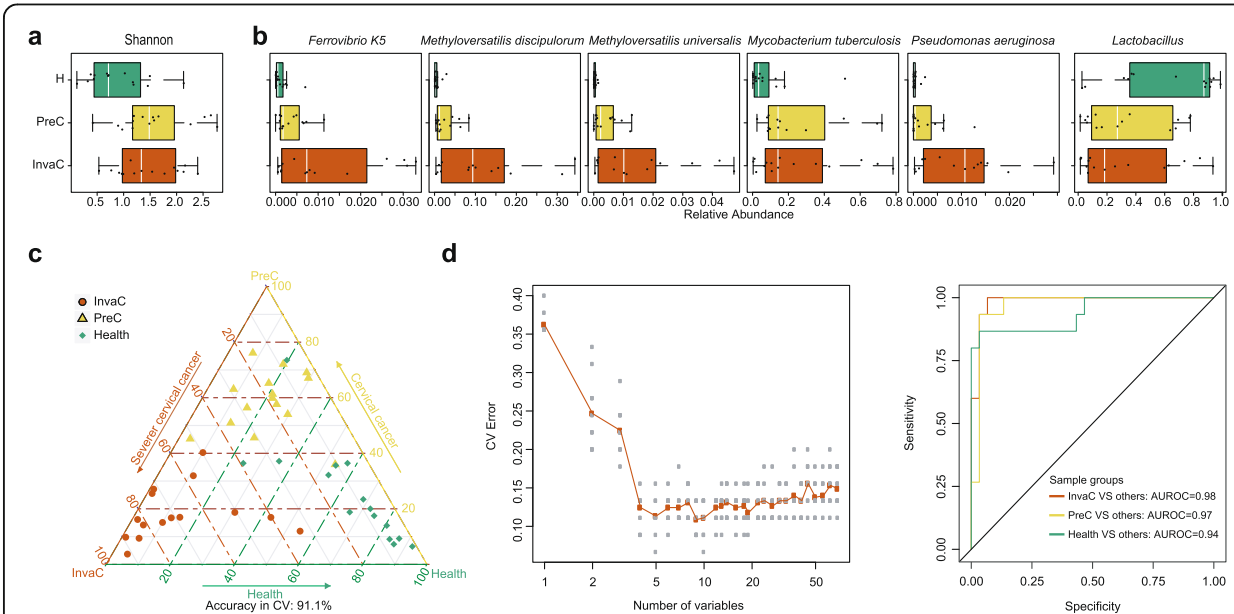

Fig. 6 Microbiome-based diagnosis of FFPE thin section from cervical cancer samples as enabled by 2bRAD-M. a Shannon and Simpson index among 15 healthy controls $(H), 15$ pre-invasive cancerous (PreC; benign), and 15 invasive cancerous (InvaC; malignant) samples. b Comparison of differential species and Lactobacillus spp. among the three groups. c The Random Forest classifier for discriminating cancer and healthy samples. In the ternary plot, each dot represents a FFPE sample. The axes indicate the microbiomebased probability of being InvaC, PreC, and H for a FFPE sample. The closer one sample is to an apex, the more likely it is predicted as to be corresponding disease states. $\mathbf{d}$ Feature selection by rebuilding Random Forest classifiers using a series of reduced sets of features. The scatter plot shows that nine variables (species) in a reduced RF model (i.e., the AUC plot on the right) can maximize model performance. And the ROC curve shows an even better discriminate performance using binary categories (averagely 0.96) 
K5 $(p$-value $=0.001)$, and Pseudomonas aeruginosa $(p$-value $=6.8 \mathrm{e}-5)($ each increase in relative abundance from $\mathrm{H}$ to PreC and to InvaC; Fig. 6b). Conversely, Lactobacillus spp. (L. paracasei, L. vaccinostercus, L. pentosus, and L. plantarum) are greatly enriched in $\mathrm{H}$ (average abundance of $63.1 \%$, in contrast to $33.9 \%$ and $32.9 \%$ in PreC and InvaC, respectively, Fig. 6b); this is consistent with a previous study [41] showing depletion of the Lactobacillales genera in "fresh," non-FFPE cervical cancerous tissues. Notably, Lactobacillus paracasei shows anticancer potential against cervix cancer cells (HeLa) in vitro [46]. Furthermore, the enrichment of Mycobacterium tuberculosis, Pseudomonas aeruginosa, and Staphylococcus aureus in PreC- and InvC-phase PPFE samples is consistent with those "fresh" tissue-based studies that reported related genus-level (via $16 \mathrm{~S}^{32}$ ) or species-level (via WMS [41, 47]) taxa. Taken as a whole, 2bRAD-M has successfully captured the microbiome structure in FFPE samples and is able to reveal previously unknown discriminative microbial features between healthy and cancerous tissues. These features may serve as potential indicative novel markers for tumor onset and progression diagnosis. To evaluate this potential, we applied Random Forest on the FFPE taxonomic profiles at the species level, and the model distinguishes $\mathrm{H}, \mathrm{PreC}$, and InvaC samples with $91.1 \%$ accuracy (tenfold cross-validation; Fig. 6c). Notably, we can achieve maximized discriminative performance ( $A U C$ 0.96) with the fewer features of the nine most important species in the RF model (Fig. 6d; Additional file 1: Table S8). Thus, 2bRAD-M offers a viable option for microbiome profiling on the vast archive of historical FFPE samples with potential application in early diagnosis and treatment of cancer.

\section{Discussion}

Using mock microbiomes plus actual samples from the stool, skin, environmentsurface, and frozen FFPE tissues, we demonstrated the ability of 2bRAD-M to profile microbiome samples that are challenging by using marker-gene or WMS sequencing approaches. Three key features are highlighted from this research. Firstly, it can analyze samples with low biomass (down to $1 \mathrm{pg}$ ), severe degradation, or high contamination. This advantage is built on the ability to (i) provide an unbiased (i.e., without the large size variation of DNA fragments) and reduced representation of metagenomes for sequencing, (ii) evenly cover all restriction sites, and (iii) generate short, iso-length reads and thus reliable quantification of tag abundance. With such advantage, 2bRAD-M is valuable to profiling microbiome samples from sparsely populated niches (e.g., indoor surfaces, blood, and skin), precious clinical specimen, or heavily degraded or contaminated tissues (e.g., FFPE sections and archeological samples). Notably, cervical cancer can be effectively cured in the preinvasive period [48], yet detection of this asymptomatic stage remains challenging which results in unnecessary delays in diagnosis and treatment [49]; therefore, 2bRAD-M sequencing of FFPE cervical cancer microbiomes, which is challenging for WMS or $16 \mathrm{~S}$ rRNA approaches, promises to turn the enormous clinical repositories of FFPE tissue specimens into a treasure trove of microbiome-driven discoveries [42].

Secondly, it can provide species-level taxonomic information for bacteria, archaea, and fungi simultaneously. The multiple species-specific 2bRAD-M tags can uniquely identify the species from the microbial community, as resolution at the "species" level instead of the "genus" level can be crucial [50]. In this case, the ability to extract the 
species-level cancer-stage-specific markers from FFPE tissues, as well as to distinguish S. epidermidis, S. hominis, and S. aureus from underarm skin samples, is crucial to the diagnosis and mechanistic understanding of inflammation [29]. Interestingly, the overall ratio of fungal and bacterial abundance, a potential indicator of ecological balance [51], is $0.31 \%$ for underarm, $0.07 \%$ for home, and $1.27 \%$ for car (significantly different), suggesting characteristic ecological features among the sites.

Finally, using 2bRAD-M, the species-level taxonomic profiling can be achieved with a much lower sequencing cost than WMS. For example, only $1 \sim 5 \%$ of the sequencing data of WMS are required by $2 \mathrm{bRAD}-\mathrm{M}$ to produce a taxonomic profile of equivalent accuracy, resulting in a cost reduction of 20-100 folds. Moreover, multi-isoRAD allows sequencing of five concatenated 2bRAD tags altogether via a single, 100-150-bp read from Illumina paired-end (PE) library [14], which further reduces the sequencing cost. The cost advantage is significant when high sequencing depth is required to address questions like unveiling the true microbial diversity or recovering rare but functionally important species in complex microbiota [25, 52]. Thus, 2bRAD-M appears especially suitable for such samples or circumstances. Notably, the performance of 2bRAD-M is limited by the availability and the potential bias of related reference genomes (e.g., 171927 bacterial and 945 archaeal genomes, yet only 293 fungal genomes in RefSeq), although the situation is rapidly improving with the steady increase of sequenced genomes for both cultured and uncultured microbes (e.g., via single-cell sequencing [53]).

\section{Conclusions}

To overcome the bottlenecks of conventional metagenomic sequencing methods, we developed a new metagenomics method (2bRAD-M) that can cost-effectively produce accurate, species-resolution, landscape-like taxonomic profiles for challenging microbiome samples that are low-biomass, high-host-contaminated, and degraded. Tests on simulated datasets, mock microbiome, and actual microbiome samples showed that 2 bRAD-M, by sequencing just about $1 \%$ of genomes, accurately generates species-level taxonomic profiles for samples that include merely 1 pg total DNA, are of $99 \%$ host DNA contamination, or consist of highly degraded fragments just $50 \mathrm{bp}$ in length. Furthermore, it can accurately reconstruct a comprehensive, species-resolution profile of bacteria, archaea, and fungi for real stool, skin, environment-surface, and FFPE samples. Therefore, 2bRAD-M greatly expands the opportunities in microbiome research in challenging settings.

\section{Materials and methods}

\section{Sample preparation and sequencing}

Mock samples

Two mock microbial communities were used to validate the stability, sensitivity, and precision of 2bRAD-M. The first consists of five evenly mixed bacterial strains including Streptococcus mutans UA159, Streptococcus agalactiae ATCC13813, Staphylococcus aureus ATCC29213, Escherichia coli DH5 $\alpha$, and Lactobacillus fermentum ATCC9338. Then, three circumstances that simulated "challenging" microbiome samples were produced (with three replicates for each sample): (1) LoA: samples with low amount DNA; a concentration gradient from $10 \mathrm{ng}$ to $1 \mathrm{pg}$ was designed, with one-tenth of the 
concentration retained each time. (2) HiD: samples with highly degraded DNA; two samples with DNA lengths about $150 \mathrm{bp}$ and $50 \mathrm{bp}$ were included. (3) HoC: samples with host DNA contamination, which consist of either $90 \%$ or $99 \%$ human DNA. To avoid the bias introduced by human DNA removal, we did not perform any depletion methods before or after DNA extraction in this study.

The second mock used in this paper is the 20-Strain Even Mix Genomic Material (named as ATCC MOCK MSA 1002, $3.87 \mathrm{ng} / \mu \mathrm{l}$; frozen $50 \mu \mathrm{l}$ in Tris- $\mathrm{HCl} \mathrm{pH}$ 8.5) which is purchased from ATCC. This mock comprises genomic DNA prepared from fully sequenced, characterized, and authenticated ATCC Genuine Cultures that were selected based on relevant phenotypic and genotypic attributes, such as Gram stain, GC content, genome size, and spore formation.

\section{Fecal and FFPE tissue samples}

The study protocol complies with the ethical guidelines of the 1975 Declaration of Helsinki, and institutional review board approval was obtained from Qingdao Institute of Bioenergy and Bioprocess Technology (QIBEBT), Chinese Academy of Sciences (CAS). Written informed consent and photography consent were obtained from each subject before enrollment. Three healthy adult individuals were enrolled as volunteers, and fecal samples were collected for deep WMS, 16S rRNA gene amplicon, and 2bRAD-M sequencing for comparison.

Three pairs of fresh and FFPE samples from healthy lung tissues of lung adenocarcinoma patients, and 45 cervical cancer-related sections in FFPE blocks (with a thickness of $4 \mu \mathrm{m}$ and an area of $3 \mathrm{~cm}^{2}$ ), underwent microbiome profiling via 2bRAD-M. The 45 cervical cancer-related sections in FFPE blocks include 15 healthy controls $(\mathrm{H}), 15$ preinvasive cancerous (PreC; benign), and 15 invasive cancerous (InvaC; malignant) ones (determined by morphological evidence of polymorphonuclear infiltration). Each of the 45 samples was from a distinct individual, i.e., a cross-sectional design. To prepare the FFPE blocks, fresh tissue samples were fixed in phosphate-buffered formalin for 24-48 $\mathrm{h}$, followed by tissue processing for $10 \mathrm{~h}$, and paraffin embedding for $20 \mathrm{~min}$ [54]. Postfixation processing of the tissues was completed in a histopathological laboratory using consistent processor protocols over years. Prior to microbiome profiling, the FFPE blocks were already stored at $17-22^{\circ} \mathrm{C}$ and $20-60 \%$ humidity levels for $1-2$ years. For these FFPE tissue sections, attempts to construct $16 \mathrm{~S}$ amplicon or WMS libraries for microbiome sequencing both failed, due to the low quality of initial DNA (Additional file 1: Fig. S8).

\section{Underarm, home, and car sampling collection protocol and processing}

For the underarm sampling protocol, individuals were subjected to a washout period where the use of anti-bacterial products was not allowed for 4-5 days. After the washout period, a 22-mm D-squame tape strip (Cuderm) was applied onto the lower underarm skin surface (without hair) using a pressure applicator. The tape strips were then pre-treated with $200 \mathrm{mg}$ of $0.1 \mathrm{~mm}$ Zirconia Silica beads and bead beat using Qiagen TissueLyser II (Valencia, CA) at $30 \mathrm{~Hz}$ for 3 min to lyse (via mechanical force) and dislodge the biomass from the tape strip. After dislodging the biomass from the tape strip, complete cell lysis is achieved via further enzymatic reaction by $2 \% \mathrm{w} / \mathrm{v}$ lysozyme, 
0.05\% w/v lysostaphin, and $1.2 \%$ Triton-X in TE buffer, followed by DNA extraction using Qiagen DNeasy Blood and Tissue Kit (Cat no. 69504, Valencia, CA). For the home and car sampling, the swab was used for wiping the surface of the cushion and carpet in the car and toy and toilet seat in the home. Specifically, wipe the swab for 20 times on an area of $4 \times 4 \mathrm{~cm}^{2}$. After collection, transfer the disposable swab to the tube containing sample storage liquid immediately and break the swab along the crease. Then, close the lid of the tube and make sure that there is no leakage. Finally, put the sample preservation solution tube in the biosafety bag for DNA extraction.

\section{DNA extraction, 16S rRNA, and shotgun metagenomic sequencing}

Genomic DNA was extracted from each fecal-containing tube using the Tissue and Blood DNA Isolation kit (Qiagen, Valencia, CA) following the manufacturer's instructions with slight modifications. PCR amplification of the V1-V3 hypervariable regions of $16 \mathrm{~S}$ rRNA genes was performed using the primer set (27F/534R) and followed the protocol developed by the Human Microbiome Project. PCR amplification reactions in triplicate for each sample were pooled at approximately equal amounts and sequenced, via the Illumina MiSeq 250 platform. All sequences were pre-processed following the standard QIIME (v.1.9) pipeline. Downstream bioinformatics analysis was performed using Parallel-Meta 3, a software package for comprehensive taxonomic and functional comparison of microbial communities. Clustering of OTUs was conducted at the $97 \%$ similarity level using a pre-clustered version of the Refseq database by BLASTN.

Paired-end metagenomic sequencing was performed for the two mock samples and fecal microbiota from three individuals via the Illumina HiSeq 2500 platform, yielding $239.73 \pm 12.34 \mathrm{~GB}$ per sample (for fecal samples, with average fragment insert size of $350 \mathrm{bp}$ and average read length of $150 \mathrm{bp}$ ). The reads were quality controlled by Trimmomatic (Sliding window 4:20; Minlength:100; MinPhred:25; Percentage of MinPhred: 80 ), and finally, $858,032,764 \pm 13,140,670$ clean reads per sample were generated and then profiled by mOTUs2 using default parameters.

\section{2bRAD-M sequencing}

The 2bRAD-M library preparation basically followed the original protocol developed by Wang et al. [13] with minor modifications. Library preparation began with the digestion of $1 \mathrm{pg}-200 \mathrm{ng}$ genomic DNA in a $15-\mu \mathrm{l}$ reaction using $4 \mathrm{U}$ BcgI $(\mathrm{NEB})$ at $37^{\circ} \mathrm{C}$ for $3 \mathrm{~h}$. Five microliters of the digested product was run on a $1 \%$ agarose gel to verify digestion. Next, ligation reaction was performed at $4{ }^{\circ} \mathrm{C}$ for $16 \mathrm{~h}$ in a $20-\mu \mathrm{l}$ volume containing $10 \mu \mathrm{l}$ of digested product, $0.2 \mu \mathrm{M}$ each of library-specific adaptors (Ada1 and Ada2), 1 mM ATP (NEB), $1 \times$ T4 DNA Ligase Buffer, and $800 \mathrm{U}$ T4 DNA ligase (NEB). Then, heat inactivation was performed for $\mathrm{BcgI}$ at $65^{\circ} \mathrm{C}$ for $20 \mathrm{~min}$.

Ligation products were amplified in 40- $\mu \mathrm{l}$ PCRs, each composed of $7 \mu$ ligated DNA, $0.1 \mu \mathrm{M}$ each primer (Primer1 and Primer2 for Illumina), $0.3 \mathrm{mM}$ dNTP, $1 \times$ Phusion HF buffer, and 0.4 U Phusion high-fidelity DNA polymerase (NEB). PCR was conducted in a DNA Engine Tetrad 2 thermal cycler (Bio-Rad) with $16-28$ cycles of $98^{\circ} \mathrm{C}$ for $5 \mathrm{~s}$, $60{ }^{\circ} \mathrm{C}$ for $20 \mathrm{~s}$, and $72{ }^{\circ} \mathrm{C}$ for $10 \mathrm{~s}$ and then a final extension of $10 \mathrm{~min}$ at $72^{\circ} \mathrm{C}$. The target band (Illumina $100 \mathrm{bp}$ ) was excised from $8 \%$ (wt/vol) polyacrylamide gel, and the DNA diffused from the gel into nuclease-free water for $12 \mathrm{~h}$ at $4{ }^{\circ} \mathrm{C}$. Finally, barcodes 
were introduced by means of PCR with platform-specific barcode-bearing primers. Forty-microliter PCR reaction contained $50 \mathrm{ng}$ of gel-extracted PCR product, $0.2 \mu \mathrm{M}$ of each primer (Primer1 and Primer3 for Illumina), $0.6 \mathrm{mM} d N T P, 1 \times$ Phusion HF buffer, and $0.8 \mathrm{U}$ Phusion high-fidelity DNA polymerase; seven cycles of the PCR profile listed above were performed. PCR products were purified by QIAquick PCR purification kit (Qiagen, Valencia, CA) and subjected to Illumina HiSeq platform sequencing. All primer and adaptor sequences are provided at Additional file 1: Table S9.

\section{Data analysis}

\section{Rationale of 2bRAD-M in overcoming high host contamination}

We attribute the ability of 2bRAD-M to overcome high host contamination to the following. (i) By sequencing the representative $1 \%$ of the human genome and microbial genomes, 2bRAD-M can dramatically reduce the cost and hence greatly increase the sequencing depth. (ii) Due to the highly imbalanced presentation of enzyme sites in microbial genomes and the human genome, microbial genomes can generate many more 2bRAD tags than the human genome. For example, if we set the 1:99 as the microbial DNA vs human DNA ratio in the mock samples (MOCK CAS), the sequencing reads ratio would change to 3:97 in the actual sequencing results. (iii) The 2bRAD fragments in the human genome are completely different from those in microbial genomes (Additional file 1: Fig. S10). Consequently, qualitative analyses such as abundance estimation for microbial species are robust against host DNA interference, and the FPs would be greatly reduced.

\section{Identification of species-specific $26 R A D-M$ markers from the most comprehensive genome database}

Firstly, a total of 173,165 microbial genomes (including bacteria, fungi, and archaea) were downloaded from the NCBI RefSeq database. Then, built-in Perl scripts (GitHub: https://github.com/shihuang047/2bRAD-M) were used to sample restriction fragments from microbial genomes by each of 16 type $2 B$ restriction enzymes, which formed a huge $2 \mathrm{bRAD}$ microbial genome database. The set of $2 \mathrm{bRAD}$ tags sampled from each genome was assigned under the GCF number, as well as GCF's taxonomic information corresponding to the whole genome. Finally, all 2bRAD tags from each GCF that occur once within the genome were compared with those of all the others. Those 2bRAD tags are specific to a species-level taxon (having no overlap with other species) were developed as species-specific 2bRAD markers, collectively forming a 2bRAD marker database. The species-specific marker database has been shown to outperform other reference databases using all $2 \mathrm{bRAD}$ tags or full genomes in taxonomic profiling (Additional file 1: Fig. S11).

\section{Simulation of 2bRAD-M sequencing data}

To test the generalizability of our 2bRAD markers for microbial profiling, we first simulated 25 abundance profiles representing microbiota from distinct habitats (gut, oral, skin, vagina, and building, with five profiles for each) with known abundance profiles (abundance was created randomly from a log-normal distribution using the function rlnorm in the R language with the following parameters: meanlog $=0$ and $s d \log =1$ ). 
Given a specified species composition (taxonomic abundance), their sequence abundance can be inferred accordingly (taxonomic abundance equals sequence abundance divided by their genome length), and then Wgsim (https:/github.com/lh3/wgsim) was used (with default parameters) to simulate the sequences. The intersection of organisms in the mOTU2, MetaPhlaAn2, and Kraken2 reference databases was used as the source genomes for the simulation. The simulation scripts for metagenomic data can be found in https://github.com/shihuang047/re-benchmarking.

\section{Usage of metagenomic profilers}

For shotgun metagenomic sequencing data, we validated the taxonomic profiles with state-of-the-arts profilers, such as Kraken2 [17], Bracken [18], mOTUs2 [19], MetaPh1An2 [20], and Centrifuge [22]. The detailed procedures are listed below.

Kraken2 (v2.0.8-beta) is a k-mer-based taxonomic classification method. It searches for 35-bp $k$-mers from the query sequence in a precomputed database that matches $k$ mers to the lowest common ancestor (LCA) taxon of all genomes that contain that taxon. The default database was constructed using complete bacterial and viral genomes from NCBI RefSeq (2019 Oct). A filtering abundance threshold of 0.01 (default) was selected. The Kraken2 command below was used: "kraken2 --threads 32 --fastq-input --gzip-compressed --paired input_1.fastq.gz input_2.fastq.gz -output output.reads -report output.report".

Bracken (v2.5) utilizes the read classification output from standard Kraken for a Bayesian re-estimation of taxonomic abundances, which significantly improves the false-positive issue of standard Kraken and implicitly normalizes for genome length. The kraken-filter was used to filter raw classifications at the 0.01 threshold. The Bracken command of "est_abundance.py - $i$ input $-k d b$-o output" was used.

MetaPhlAn2 (2.96.1) is a marker-gene alignment approach that relies on a precomputed database containing clade-specific marker genes. Query reads are aligned via bowtie 2 to the marker genes for microbial identification and abundance estimation. The database version used is mpa v296 CHOCOPhlAn 201901. The MetaPhlAn2 command of "metaphlan2.py input_1.fastq.gz, input_2.fastq.gz --input_type fastq --nproc threads --bowtie2out output_bowtie2out.txt -o output.report" was used.

mOTUs2 (v2.5.1, database version 2.5.0) is a marker-based method that compiles a large variety of phylogenetic marker genes from multiple biomes. Query reads are aligned using bwa mem and further processed to generate an abundance profile. The mOTUs2 command of "motus profile -f input_1.fastq.gz -r input_2.fastq.gz -o output.report - $t$ threads" was used.

Centrifuge (v1.0.3-beta) is a microbial classification engine based on FM-index which enables rapid, accurate, and sensitive taxonomic labeling of short reads. To analyze 2bRAD sequencing data of MOCK CAS (Fig. 3a), we employed Centrifuge to perform the 2bRAD-tag-level taxonomic classification that none of other existing profilers can handle. We started by customizing a database for Centrifuge that only contains genomes of the five species in MOCK CAS. Next, Centrifuge was used as a search engine to calculate the 2bRAD read-level proportion of five microbial species. Accordingly, we repeated this process using WMS for benchmarking purpose. 


\section{Calculation of relative abundance in $26 R A D-M$}

Firstly, to identify microbial species within each sample, all sequenced 2bRAD tags after quality control were mapped (using a built-in Perl script) against the 2bRAD marker database which contains all 2bRAD tags theoretically unique to each of 26,163 microbial species in RefSeq database (Fig. 1). To control the false-positive in the species identification, a $G$ score was derived for each species identified within a sample as below, which is a geometric mean of the proportion of the species-specific markers that have been captured (by sequencing) and the number of all detected species-specific markers (by sequencing) of this species. The $G$ score is more sensitive as the threshold than the relative abundance used in conventional WMS (Additional file 1: Fig. S12). The threshold of $G$ score for a false-positive discovery of microbial species was set as 10 .

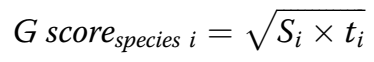

$S$ : the number of reads assigned to all 2bRAD markers belonging to species $i$ within a sample.

$t$ : number of all 2bRAD markers of species $i$ that have been sequenced within a sample

We estimated the relative abundance of each microbial species in a sample using the formula as below. We first calculated the average read coverage of all 2bRAD markers for each species, which represent the number of individuals belonging to a species present in a sample at a given sequencing depth. The relative abundance of a given species is then calculated as the ratio of the number of microbial individuals belonging to a species against the total number of individuals from known species that can be detected within a sample, with the default $G$ score of 10 .

$$
\text { Relative abundance }_{\text {species } i}=\frac{S_{i} / T_{i}}{\sum_{i=1}^{n} S_{i} / T_{i}}
$$

$S$ : the number of reads assigned to all 2bRAD markers of species $i$ within a sample $T$ : the number of all theoretical 2bRAD markers of species $i$

\section{Calculation of precision, recall, L2 similarity, and Pearson coefficient}

For overall performance assessment, we applied precision and recall to evaluate the accuracy of microbial identification, while L2 distance [16] was employed to evaluate the accuracy of abundance estimation within a sample. Precision is the proportion of true positive species against the total number of species identified by a method, whereas recall is defined as the proportion of true positive species against the number of all species actually existing in a sample. To evaluate the accuracy of abundance profiles, we calculate the L2 distance between ground-truth abundance profile to each of the taxonomic profiles at a given taxonomic level (e.g., species or genus) produced by metagenomic sequencing methods. To visualize the benchmarking results more intuitively, we further employed L2 similarity calculated as the 1-L2 distance for performance comparisons. As for Shannon correlation, it is denoted by the difference between 1 and the absolute value of 1 minus the ratio of the predicted Shannon index and its ground truth. In addition, in Pearson correlation analysis, for those unique species that are identified only in one method, we replaced their abundance with 0 in calculating the Pearson correlation coefficient. 


\section{Diagnosis model of cancer samples}

Random Forest models were trained to identify cancer status using the taxonomy profiles on the species level. Default parameters of the $\mathrm{R}$ implementation of algorithm were applied ( $\mathrm{R}$ package 'randomForest', ntree $=5000$, using default mtry of $p / 3$ where $p$ is the number of input taxa). The performance of RF models based on microbiota was evaluated with a tenfold cross-validation approach.

Additional details are provided in Additional file 1: Supplemental Methods.

\section{Supplementary Information}

The online version contains supplementary material available at https://doi.org/10.1186/s13059-021-02576-9.

Additional file 1. Supplemental Methods, Figures S1-S12, Tables S1-S9

Additional file 2. Additional data files

Additional file 3. Review history

\section{Acknowledgements}

We thank Dr. Rob Knight and Dr. Yoshiki Vázquez-Baeza from UCSD for the helpful discussion on technical issues. This work was funded by Grant 32030003 from National Natural Science Foundation (NSFC), 2018YFC310802 from National Key Research and Development Program of China, XDPB1804-1 from the Strategic Priority Research Program of the CAS, 31800088 from NSFC, 2019 M652501 from the Postdoctoral Science Foundation of China, SKJC-KJ-2019KY01 from the Sanya Yazhouwan Science and Technology City Management Foundation. S. W. and J. X. acknowledges the support by the Taishan Scholar Fund of Shandong Province of China.

\section{Peer review information}

Andrew Cosgrove and Barbara Cheifet were the primary editors of this article and managed its editorial process and peer review in collaboration with the rest of the editorial team.

\section{Review history}

The review history is available as Additional file 3.

\section{Authors' contributions}

Z.S. and S.H. conceived of the study. S.W. and J.X. designed the experiments and supervised the study. P.Z., J.L., L.Z, Q.N., and X.W carried out the experiments. Z.S., S.H., S.W., and J.X. wrote the manuscript with support from L.T., H.Z., Z.B., and J.L.. Z.S., G.J., and M.Z. planned and carried out the simulations. Z.S., S.H., and R.Z. designed the model and the computational framework and analyzed the data. The authors read and approved the final manuscript.

\section{Availability of data and materials}

The sequencing data of 16S, WMS, and 2bRAD-M for MOCK (MOCK CAS and ATCC MOCK MSA 1002) and FFPE (Only 2bRAD-M sequencing data) were submitted to figshare: https://doi.org/10.6084/m9.figshare.12272360.v8. The sequencing data of 165 and 2bRAD-M for typical low-biomass samples (underarm, home, and car) and fecal samples were submitted to https://doi.org/10.6084/m9.figshare.13514960.v4. All the above sequencing data were also submitted to SRA (SRP300672) under PRJNA689204 in NCBI [55] as well as the ultra-deep WMS data of stool samples. The 2bRAD-M computational pipeline and related database files are publicly available at Github [56]: https://github.com/shihuang047/2bRAD-M. The computational pipeline of 2bRAD-M is licensed under the MIT license. All source data and codes for the generation of figures and tables in the manuscript can be downloaded from: https://github.com/shihuang047/2bRAD-M-manuscript.

\section{Declarations}

Ethics approval and consent to participate

The study protocol complies with the ethical guidelines of the 1975 Declaration of Helsinki, and institutional review board approval was obtained from Qingdao Institute of Bioenergy and Bioprocess Technology (QIBEBT), Chinese Academy of Sciences (CAS). Written informed consent and photography consent were obtained from each subject before enrollment.

\section{Competing interests}

Rongchao Zhang, Lisha Zhou, Qianya Niu, and Xiuping Wang are affiliated to Qingdao OE Biotechnology Company. Lam Tzehau, Helen Zhao, and Jiquan Liu are affiliated with Procter \& Gamble Company. The remaining authors declare that the research was conducted in the absence of any commercial or financial relationships that could be construed as a potential conflict of interest.

\section{Author details}

${ }^{1}$ Single-Cell Center, CAS Key Laboratory of Biofuels and Shandong Key Laboratory of Energy Genetics, Qingdao Institute of BioEnergy and Bioprocess Technology, Chinese Academy of Sciences, Qingdao, China. ${ }^{2}$ Channing Division of Network Medicine, Brigham and Women's Hospital and Harvard Medical School, Boston, USA. ${ }^{3}$ University of Chinese Academy of Sciences, Beijing, China. ${ }^{4}$ UCSD Health Department of Pediatrics and Center for Microbiome Innovation at 
Jacobs School of Engineering, University of California, San Diego, San Diego, USA. ${ }^{5}$ Faculty of Dentistry, The University of Hong Kong, Hong Kong SAR, China. ${ }^{6}$ Procter \& Gamble Singapore Innovation Center, Singapore, Singapore. ${ }^{7}$ Sars-Fang Centre \& MOE Key Laboratory of Marine Genetics and Breeding, Ocean University of China, Qingdao, Qingdao, China. ${ }^{8}$ Qingdao OE Biotechnology Company Limited, Qingdao, China. ${ }^{9}$ Key Laboratory of Dairy Biotechnology and Engineering, Ministry of Education, Inner Mongolia Agricultural University, Hohhot, China. ${ }^{10}$ Qingdao National Laboratory of Marine Science and Technology, Qingdao, China. ${ }^{11}$ Laboratory of Tropical Marine Germplasm Resources and Breeding Engineering, Sanya Oceanographic Institution, Ocean University of China, Sanya, China.

Received: 8 February 2021 Accepted: 14 December 2021

Published online: 26 January 2022

\section{References}

1. Knight R, Vrbanac A, Taylor BC, Aksenov A, Callewaert C, Debelius J, et al. Best practices for analysing microbiomes. Nat Rev Microbiol. 2018;16(7):410-22. https://doi.org/10.1038/s41579-018-0029-9.

2. Cho I, Blaser MJ. The human microbiome: at the interface of health and disease. Nat Rev Genet. 2012;13(4):260-70. https://doi.org/10.1038/nrg3182.

3. Franzosa EA, Hsu T, Sirota-Madi A, Shafquat A, Abu-Ali G, Morgan XC, et al. Sequencing and beyond: integrating molecular 'omics' for microbial community profiling. Nat Rev Microbiol. 2015;13(6):360-72. https://doi.org/10.1038/ nrmicro3451.

4. Quince C, Walker AW, Simpson JT, Loman NJ, Segata N. Shotgun metagenomics, from sampling to analysis. Nat Biotechnol. 2017:35(9):833-44. https://doi.org/10.1038/nbt.3935.

5. Davey JW, Hohenlohe PA, Etter PD, Boone JQ, Catchen JM, Blaxter ML. Genome-wide genetic marker discovery and genotyping using next-generation sequencing. Nat Rev Genet. 2011;12(7):499-510. https://doi.org/10.1038/nrg3012.

6. Andrews KR, Good JM, Miller MR, Luikart G, Hohenlohe PA. Harnessing the power of RADseq for ecological and evolutionary genomics. Nat Rev Genet. 2016;17(2):81-92. https://doi.org/10.1038/nrg.2015.28.

7. Grewe F, Huang J-P, Leavitt SD, Lumbsch HT. Reference-based RADseq resolves robust relationships among closely related species of lichen-forming fungi using metagenomic DNA. Sci Rep. 2017;7(1):9884. https://doi.org/10.1038/s41 598-017-09906-7.

8. Liu M, Worden P, Monahan LG, DeMaere MZ, Burke CM, Djordjevic SP, et al. Evaluation of ddRAD seq for reduced representation metagenome sequencing. Peerj. 2017;5:e3837. https://doi.org/10.7717/peerj.3837.

9. Holmes I, Rabosky ARD. Natural history bycatch: a pipeline for identifying metagenomic sequences in RADseq data. Peerj. 2018;6:e4662. https://doi.org/10.7717/peeri.4662.

10. Hess MK, Rowe SJ, Van Stijn TC, Henry HM, Hickey SM, Brauning R, et al. A restriction enzyme reduced representation sequencing approach for low-cost, high-throughput metagenome profiling. PLoS One. 2020;15(4):e0219882. https://doi. org/10.1371/journal.pone.0219882.

11. Davey JW, Cezard T, Fuentes-Utrilla P, Eland C, Gharbi K, Blaxter ML. Special features of RAD sequencing data: implications for genotyping. Mol Ecol. 2013;22(11):3151-64. https://doi.org/10.1111/mec.12084.

12. Aird D, Ross MG, Chen WS, Danielsson M, Fennell T, Russ C, et al. Analyzing and minimizing PCR amplification bias in Illumina sequencing libraries. Genome Biol. 2011;12(2):R18. https://doi.org/10.1186/gb-2011-12-2-r18.

13. Wang S, Meyer E, McKay JK, Matz MV. 2b-RAD: a simple and flexible method for genome-wide genotyping. Nat Methods. 2012;9:808.

14. Wang S, Liu P, LV J, Li Y, Cheng T, Zhang L, et al. Serial sequencing of isolength RAD tags for cost-efficient genomewide profiling of genetic and epigenetic variations. Nat Protoc. 2016;11(11):2189-200. https://doi.org/10.1038/nprot.201 6.133.

15. Wang S, LV J, Zhang L, Dou J, Sun Y, Li X, et al. MethylRAD: a simple and scalable method for genome-wide DNA methylation profiling using methylation-dependent restriction enzymes. Open Biol. 2015;5(11):150130. https://doi.org/1 $0.1098 /$ rsob.150130.

16. Ye SH, Siddle KJ, Park DJ, Sabeti PC. Benchmarking metagenomics tools for taxonomic classification. Cell. 2019;178(4): 779-94. https://doi.org/10.1016/j.cell.2019.07.010.

17. Wood DE, Lu J, Langmead B. Improved metagenomic analysis with Kraken 2. Genome Biol. 2019;20(1):257. https://doi. org/10.1186/s13059-019-1891-0.

18. Lu J, Breitwieser FP, Thielen P, Salzberg SL. Bracken: estimating species abundance in metagenomics data. Peerj Comput Sci. 2017;3:e104. https://doi.org/10.7717/peerj-cs.104.

19. Milanese A, Mende DR, Paoli L, Salazar G, Ruscheweyh H-J, Cuenca M, et al. Microbial abundance, activity and population genomic profiling with mOTUs2. Nat Commun. 2019;10(1):1014. https://doi.org/10.1038/s41467-019-08844-4.

20. Truong DT, Franzosa EA, Tickle TL, Scholz M, Weingart G, Pasolli E, et al. MetaPhlAn2 for enhanced metagenomic taxonomic profiling. Nature Methods. 2015;12(10):902-3. https://doi.org/10.1038/nmeth.3589.

21. Sun Z, Huang S, Zhang M, Zhu Q, Haiminen N, Carrieri AP, et al. Challenges in benchmarking metagenomic profilers. Nat Methods. 2021;18(6):618-26. https://doi.org/10.1038/s41592-021-01141-3.

22. Kim D, Song L, Breitwieser FP, Salzberg SL. Centrifuge: rapid and sensitive classification of metagenomic sequences. Genome Res. 2016;26(12):1721-9. https://doi.org/10.1101/gr.210641.116.

23. Amos GCA, Logan A, Anwar S, Fritzsche M, Mate R, Bleazard T, et al. Developing standards for the microbiome field. Microbiome. 2020;8(1):98. https://doi.org/10.1186/s40168-020-00856-3.

24. Hillmann B, Al-Ghalith GA, Shields-Cutler RR, Zhu Q, Gohl DM, Beckman KB, et al. Evaluating the information content of shallow shotgun metagenomics. mSystems. 2018;3(6):e00069-18. https://doi.org/10.1128/mSystems.00069-18.

25. Zaheer R, Noyes N, Ortega Polo R, Cook SR, Marinier E, Van Domselaar G, et al. Impact of sequencing depth on the characterization of the microbiome and resistome. Sci Rep. 2018;8(1):5890. https://doi.org/10.1038/s41598-018-24280-8.

26. de Goffau MC, Lager S, Salter SJ, Wagner J, Kronbichler A, Charnock-Jones DS, et al. Recognizing the reagent microbiome. Nat Microbiol. 2018;3(8):851-3. https://doi.org/10.1038/s41564-018-0202-y. 
27. Lam TH, Verzotto D, Brahma $P, N g A H Q, H u P$, Schnell $D$, et al. Understanding the microbial basis of body odor in prepubescent children and teenagers. Microbiome. 2018;6(1):213. https://doi.org/10.1186/s40168-018-0588-z.

28. James AG, Austin CJ, Cox DS, Taylor D, Calvert R. Microbiological and biochemical origins of human axillary odour. FEMS Microbiol Ecol. 2013;83(3):527-40. https://doi.org/10.1111/1574-6941.12054.

29. Nakatsuji T, Chen TH, Narala S, Chun KA, Two AM, Yun T, et al. Antimicrobials from human skin commensal bacteria protect against Staphylococcus aureus and are deficient in atopic dermatitis. Sci Transl Med. 2017;9:eaah4680.

30. Lax S, Smith DP, Hampton-Marcell J, Owens SM, Handley KM, Scott NM, et al. Longitudinal analysis of microbial interaction between humans and the indoor environment. Science. 2014;345(6200):1048-52. https://doi.org/10.1126/ science.1254529.

31. Adams RI, Miletto M, Taylor JW, Bruns TD. The diversity and distribution of fungi on residential surfaces. PLoS One. 2013; 8(11):e78866. https://doi.org/10.1371/journal.pone.0078866.

32. Saunders CW, Scheynius A, Heitman J. Malassezia fungi are specialized to live on skin and associated with dandruff, eczema, and other skin diseases. PLoS Pathog. 2012;8(6):e1002701. https://doi.org/10.1371/journal.ppat.1002701.

33. Rocchi S, Reboux G, Scherer E, Laboissière A, Zaros C, Rouzet A, et al. Indoor microbiome: quantification of exposure and association with geographical location, meteorological factors, and land use in France. Microorganisms. 2020;8(3): 341. https://doi.org/10.3390/microorganisms8030341.

34. Sharpe RA, Thornton CR, Tyrrell J, Nikolaou V, Osborne NJ. Variable risk of atopic disease due to indoor fungal exposure in NHANES 2005-2006. Clin Exp Allergy. 2015;45(10):1566-78. https://doi.org/10.1111/cea.12549.

35. Greaves MW, Camp RD. Prostaglandins, leukotrienes, phospholipase, platelet activating factor, and cytokines: an integrated approach to inflammation of human skin. Arch Dermatol Res. 1988;280 Suppl:S33-41.

36. Gallo RL. S. epidermidis influence on host immunity: more than skin deep. Cell Host Microbe. 2015;17(2):143-4. https:// doi.org/10.1016/j.chom.2015.01.012.

37. Leinwand JC, Miller G. Microbes as biomarkers and targets in pancreatic cancer. Nat Rev Clin Oncol. 2019;16(11):665-6. https://doi.org/10.1038/s41571-019-0276-3.

38. Geller LT, Barzily-Rokni M, Danino T, Jonas OH, Shental N, Nejman D, et al. Potential role of intratumor bacteria in mediating tumor resistance to the chemotherapeutic drug gemcitabine. Science. 2017;357:1156.

39. Guglielmi G. Gut microbes join the fight against cancer. Nature. 2018;557(7706):482-4. https://doi.org/10.1038/d41586-01 8-05208-8.

40. Nejman D, Livyatan I, Fuks G, Gavert N, Zwang Y, Geller LT, et al. The human tumor microbiome is composed of tumor type-specific intracellular bacteria. Science. 2020;368(6494):973-80. https://doi.org/10.1126/science.aay9189.

41. Klein C, Gonzalez D, Samwel K, Kahesa C, Mwaiselage J, Aluthge N, et al. Relationship between the cervical microbiome, HIV status, and precancerous lesions. mBio. 2019;10(1):e02785-18.

42. Poore GD, Kopylova E, Zhu Q, Carpenter C, Fraraccio S, Wandro S, et al. Microbiome analyses of blood and tissues suggest cancer diagnostic approach. Nature. 2020;579:567.

43. Walker SP, Tangney M, Claesson MJ. Sequence-based characterization of intratumoral bacteria-a guide to best practice. Front Oncol. 2020;10. https://doi.org/10.3389/fonc.2020.00179.

44. Pinto-Ribeiro I, Ferreira RM, Pereira-Marques J, Pinto V, Macedo G, Carneiro F, et al. Evaluation of the use of formalinfixed and paraffin-embedded archive gastric tissues for microbiota characterization using next-generation sequencing. Int J Mol Sci. 2020;21(3). https://doi.org/10.3390/ijms21031096.

45. Do H, Dobrovic A. Sequence artifacts in DNA from formalin-fixed tissues: causes and strategies for minimization. Clin Chem. 2015;61 (1):64-71. https://doi.org/10.1373/clinchem.2014.223040.

46. Riaz Rajoka MS, Zhao H, Lu Y, Lian Z, Li N, Hussain N, et al. Anticancer potential against cervix cancer (HeLa) cell line of probiotic Lactobacillus casei and Lactobacillus paracasei strains isolated from human breast milk. Food Funct. 2018;9(5): 2705-15. https://doi.org/10.1039/C8FO00547H.

47. Johansen IS, Thomsen VO, Forsgren A, Hansen BF, Lundgren B. Detection of Mycobacterium tuberculosis complex in formalin-fixed, paraffin-embedded tissue specimens with necrotizing granulomatous inflammation by strand displacement amplification. J Mol Diagn. 2004;6(3):231-6. https://doi.org/10.1016/S1525-1578(10)60515-6.

48. Patel A. Benign vs malignant tumors. JAMA Oncology. 2020;6(9):1488. https://doi.org/10.1001/jamaoncol.2020.2592.

49. Fontaine PL, Saslow D, King VJ. ACS/ASCCP/ASCP guidelines for the early detection of cervical cancer. Am Fam Physician. 2012;86(501):506-7.

50. Reese AT, Dunn RR. Drivers of microbiome biodiversity: a review of general rules, feces, and ignorance. mBio. 2018;9(4): e01294-18.

51. Wagg C, Schlaeppi K, Banerjee S, Kuramae EE, van der Heijden MGA. Fungal-bacterial diversity and microbiome complexity predict ecosystem functioning. Nat Commun. 2019;10(1):4841. https://doi.org/10.1038/s41467-019-12798-y.

52. Pereira-Marques J, Hout A, Ferreira RM, Weber M, Pinto-Ribeiro I, van Doorn L, et al. Impact of host DNA and sequencing depth on the taxonomic resolution of whole metagenome sequencing for microbiome analysis. Front Microbiol. 2019;10:1277. https://doi.org/10.3389/fmicb.2019.01277.

53. Lasken RS, McLean JS. Recent advances in genomic DNA sequencing of microbial species from single cells. Nat Rev Genet. 2014;15(9):577-84. https://doi.org/10.1038/nrg3785.

54. Rahmadwati NG, Ros M, Todd C. Morphological characteristics of cervical cells for cervical cancer diagnosis. In: Gaol FL, Nguyen QV, editors. Proceedings of the 2011 2nd International Congress on Computer Applications and Computational Science; 2012//; Berlin, Heidelberg. Berlin Heidelberg: Springer; 2012. p. 235-43.

55. Sun Z, Huang S, Zhu P, Tzehau L, Zhao H, Lv J, Zhang R, Zhou L, Niu Q, Wang X, et al: Datasets. National Center for Biotechnology Information. http://www.ncbinlm.nih.gov/Traces/study/?acc=PRJNA689204\%20\&0=acc_5\%3Aa (2021).

56. Sun Z, Huang S, Zhu P, Tzehau L, Zhao H, Lv J, Zhang R, Zhou L, Niu Q, Wang X, et al: 2bRAD-M computational pipeline. Github. https://github.com/shihuang047/2bRAD-M (2021).

\section{Publisher's Note}

Springer Nature remains neutral with regard to jurisdictional claims in published maps and institutional affiliations. 